\title{
STM Imaging of Photochromic Spiropyrans
}

\author{
L. Zheng, M. C. Biewer, and I. H. Musselman \\ Department of Chemistry, University of Texas at Dallas, Richardson, TX 75083-0688
}

Scanning tunneling microscopy (STM) has been successfully applied to the observation of organic adsorbates at the liquid/solid interface with molecular resolution [1-3]. Recent research in this field has focused on developing STM into a chemically sensitive tool including identification of functional groups within the adsorbed molecule [4-10]. Among numerous image contrast mechanisms that have been proposed, the electronic/geometric mechanism is of major consideration $[1,2,6-8]$. The electronic factor is related to the coupling between the energy levels of the adsorbate and the surface Fermi level while the geometric factor addresses the spatial overlap between the STM tip and the functional group. STM image interpretation, especially of molecules with multiple aromatic rings and of biological molecules, continues to be a challenge.

Previous STM studies in our laboratory of para-substituted phenyloctadecyl ethers (X-POEs, where $\mathrm{X}=\mathrm{H}, \mathrm{Cl}, \mathrm{Br}, \mathrm{I}, \mathrm{CN}, \mathrm{NO}_{2}, \mathrm{NH}_{2}$ ) and hydroxy-terminated para-substituted phenylhexadecyl ethers (X-PHEs, where $\mathrm{X}=\mathrm{CN}, \mathrm{NO}_{2}, \mathrm{NH}_{2}$ ) physisorbed onto highly oriented pyrolytic graphite (HOPG) suggested that the image contrast mechanism involved resonance tunneling through molecular orbitals of the adsorbate [5,6]. The bias-dependent contrast observed in the STM images resembled calculated electron density contours of bonding molecular orbitals [5,6,9,10]. The HOMOresembling images of X-POEs that decoupled the electronic factor from the geometric one enabled quantitative discrimination of the functional groups through the analysis of image contrast $[6,10]$. The orientation of the functional group in a lamella and relative to the HOPG substrate significantly influenced its contrast [10].

In this project, a class of photochromic compounds X-TSPs (benzothiozoline-spiropyran with an octadecyloxy group at the 6' position, where $\mathrm{X}=\mathrm{I}, \mathrm{Br}, \mathrm{NO}_{2}, \mathrm{FIG}$. 1) was studied to further explore the image contrast of functional groups as well as that of heterocyclic rings compared to the phenyl ring in X-POEs. X-TSPs are photochromic compounds which convert between an open conjugated merocyanine and a closed spiropyran form. The two forms can be interconverted with excitation of an appropriate wavelength of light. In polar solvents, the merocyanine form is the ground-state stabilized form. Bias-dependent STM images of X-TSPs were acquired between -300 and -1200 $\mathrm{mV}$ (FIG. 2). The molecules were packed with alkyl tails fully interdigitated and aromatic rings head-to-head and offset. After high-resolution HOMO-resembling images are acquired, the intensities of functional groups can be compared with values already measured for the X-POEs. Also of interest is the variation in contrast for different rings in one X-TSP molecule as well as the change in ring contrast owing to different substituents $\left(\mathrm{I}, \mathrm{Br}, \mathrm{NO}_{2}\right)$. The data can be used to illustrate the stabilization of the resonance form by altering functionality, which is conventionally studied in solution. This is the first time that a photochromic spiropyran has been imaged by STM and that an image contrast study has been applied to a multi-ring system.

References

[1] L. C. Giancarlo and G. W. glynn, Annu. Rev. Phys. Chem. 49 (1998) 297.

[2] R. Lazzaroni et al., J. Chem. Phys. 107 (1997) 99. 
[3] K. Kim and A. J. Matzger, J. Am. Chem. Soc. 124 (2002) 8772.

[4] T. Nishino et al., Phys. Chem. Chem. Phys. 3 (2001) 1867.

[5] H. S. Lee et al., Langmuir 14 (1998) 7475.

[6] H. S. Lee et al., Anal. Chem. 73 (2001) 5532.

[7] L. Giancarlo et al., Langmuir 14 (1998) 1465.

[8] C. L. Claypool et al., J. Phys. Chem. B 103 (1999) 7077.

[9] K. Kangasniemi, Synthesis and STM Imaging of Para Substituted Phenylethers M.S. Thesis, UTDallas, 2001.

[10] A. J. M. Lubag, Jr., Synthesis and STM Imaging of Substituted Phenyl Alkyl Ethers: Towards Functional Group Resolution M.S. Thesis, UT-Dallas, 2002.

[11] The support of this research by the Robert A. Welch Foundation (IHM, MCB) is gratefully acknowledged.

A

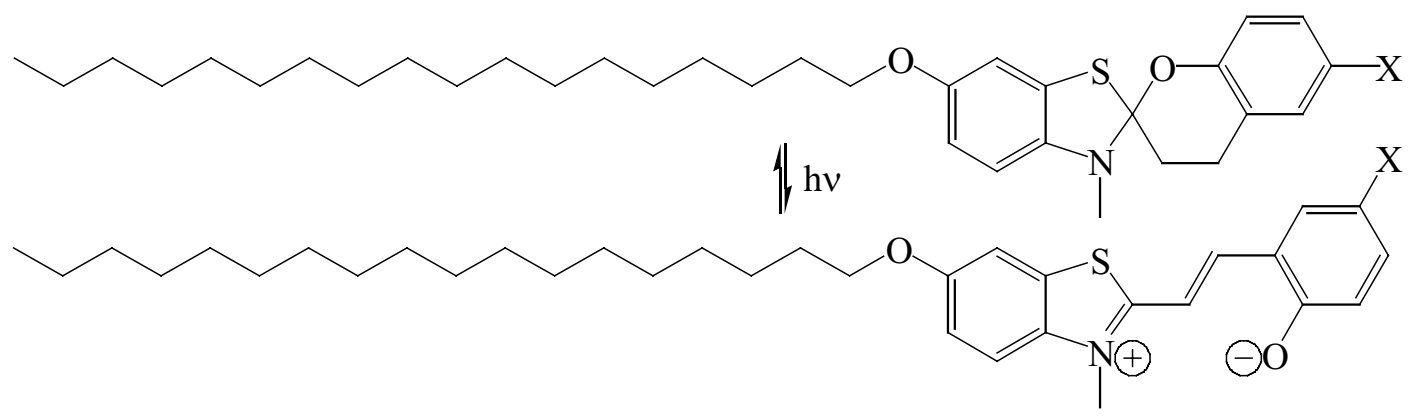

FIG. 1. Structure of X-TSP (A. spiropyran form, B. merocyanine form)

A

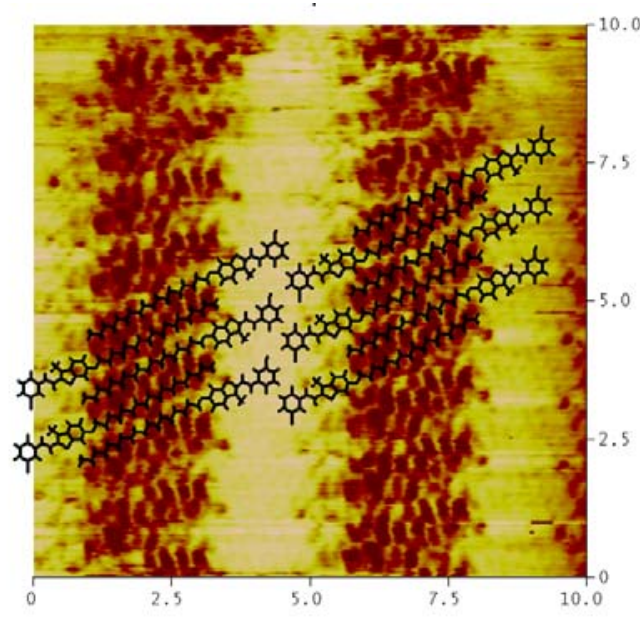

B

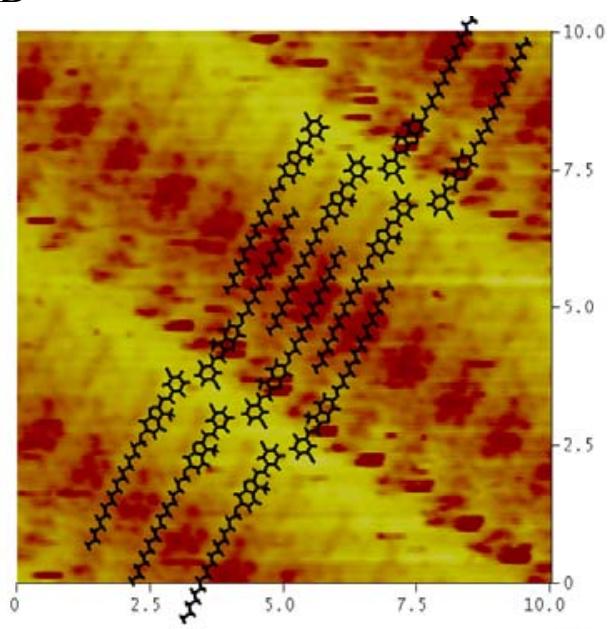

FIG. 2. STM images $\left(10 \times 10 \mathrm{~nm}^{2}\right)$ of I-TSP ${ }^{n \mathrm{~nm}}$ with HyperChem ${ }^{\circledR}$ molecular models $(A$. bias $=-600$ $\mathrm{mV}$, B. bias $=-1000 \mathrm{mV}$ ) 